\title{
Perfis Diferenciais de Perda de Memória entre a Demência Frontotemporal e a do Tipo Alzheimer
}

\author{
Ricardo F. Allegri ${ }^{1,2}$ \\ Paula Harris \\ Cecília Serrano \\ Universidade de Buenos Aires, Argentina \\ Nélson Delavald \\ Universidade Federal do Rio Grande do Sul
}

\begin{abstract}
Resumo
Os estados iniciais da demência tipo Alzheimer (DTA) caracterizam-se classicamente por deterioração da memória enquanto que as mudanças de conduta e de personalidade aparecem nas etapas iniciais da demência frontotemporal (DFT). Entretanto, na prática clínica, o diagnóstico diferencial é difícil. O objetivo do presente trabalho foi estudar o rendimento da memória de pacientes com DTA $(n=20)$ e com DFT $(n=20)$ comparando-o com um grupo de controles $(n=20)$. Os pacientes, emparelhados por idade e escolaridade, foram avaliados com uma bateria neuropsicológica exaustiva. Para a avaliação da memória, examinouse a "queixa subjetiva" de perda de memória (memória subjetiva), a aprendizagem de uma lista de palavras (memória episódica) e o desempenho no teste de denominação de Boston (memória semântica). As pontuações de ambos os grupos de pacientes, na grande maioria das provas, foram significativamente inferiores às dos controles. Os pacientes com DTA mostraram uma deterioração global da memória episódica (tipo amnésia) e semântica com um alto nível de queixa subjetiva. Os sujeitos com DFT, por outro lado, apresentaram um déficit de memória importante na recuperação da informação, mas com melhores capacidades de registro da informação, apesar das dificuldades de reconhecimento do seu distúrbio.

Palavras-chave: Alzheimer; memória; queixa subjetiva; demência frontotemporal; neuropsicologia.
\end{abstract}

Differential Memory Profile Between Frontotemporal Dementia and Alzheimer's Disease

\begin{abstract}
While in Dementia of Alzheimer's Type (DAT) the initial states are characterized by the deterioration of memory, in frontotemportal dementia (FTD) the early symptoms are basically changes in behavior. However, in daily clinical practice, the establishment of a differential diagnosis is very difficult to be found. The objective of the present work is to compare the memory efficiency of patients with DTA $(n=20)$ and FTD $(n=20)$ to a control group. The patients, matched for age and educational level, were evaluated with basis on a number of exhausting neuropsychology tests. In order to examine memory, we used the learning, free recall and recognition of a list of words (episodic memory), the analysis of the performance in the Boston Naming Test (semantic memory) and the subjects memory complaints (subjective memory). The scores obtained on the most part of the tests by the two groups (DAT and FTD) were significantly worse than those of the control group. DAT patients, on the other hand, showed an important memory deficit on free recall but less encoding capacities, despite the difficulties in acknowledging their disturbance. Memory profile of FTD patients was characterized by forgetfulness while DAT profile was an amnesic one.

Keywords: Alzheimer; memory; complaint; frontotemporal dementia; neuropsychology.
\end{abstract}

As síndromes demenciais podem ser classificadas basicamente em duas categorias etiológicas, a saber: degenerativas e não-degenerativas. As demências nãodegenerativas decorrem de acidentes vasculares, processos infecciosos, traumatismos, deficiências nutricionais,

${ }^{1}$ Endereço para correspondência: Servicios de Neurología y de Neuropsicologia, Cemic, CONICET, Galvan 4102 (1431), Buenos Aires, Argentina. Fax: (54-11) 4922-9833. E-mail: rallegri@fibertel.com.ar

${ }^{2}$ CONICET (Concelho Nacional de Investigações Científicas e Tecnológicas-Argentina) contaminações tóxicas, tumores ou outros. Os processos demenciais degenerativos, por sua vez, podem ter uma origem predominantemente cortical, como a enfermidade de Alzheimer, ou subcortical, como a enfermidade de Huntington. Esta divisão entre demências corticais e subcorticais baseia-se na localização lesional predominante da enfermidade em questão e implica padrões específicos de transtorno cognitivo (Dewer, 1995).

Dentre as demências degenerativas corticais, a enfermidade de Alzheimer tem maior freqüência estatística 
(Almeida \& Nitrini, 1995; Cummings \& Benson, 1992; Mortimer,1983; Neary e cols., 1986). Outros tipos de demência degenerativa cortical, não obstante, são diagnosticados, ainda que com uma freqüência muito menor. Esta baixa incidência de diagnósticos de demência degenerativa não-Alzheimer deve-se, em parte, ao desconhecimento clínico acerca destas patologias e também devido a um sobrediagnóstico da síndrome de Alzheimer. Em outras palavras, muitos pacientes diagnosticados como tendo a Doença de Alzheimer, na realidade apresentam quadros clínicos das demais demências corticais. Em exames pós-óbito realizados em pacientes diagnosticados com demência degenerativa, encontra-se uma freqüência de $15 \%$ a $25 \%$ de demências não-Alzheimer, sendo que, em muitos destes casos, o paciente havia recebido um diagnóstico clínico de demência Alzheimer (Pachana, Bonne, Miller, Cummings \& Berman, 1996). A quase totalidade dos casos diagnosticados em exames pós-óbito como sendo nãoAlzheimer, constitui o quadro das demências frontotemporais (DFT), cujos subtipos são: afasia progressiva primária (APP), demência semântica (DS) e demências frontotemporais (DFT).

No subtipo designado afasia progressiva primária (APP) incluem-se pacientes que começam, de forma insidiosa e progressiva, com um déficit de linguagem, o qual se mantém como o único transtorno cognitivo durante pelo menos dois anos (Duyckaerts, He, Seilhean, \& Hauw, 1995; Mesulam, 1982). Na APP, a afasia apresenta-se isolada e progride sem os componentes habituais de cognição e conduta das demências. Os pacientes com APP obtêm pontuações normais ou às vezes superiores nos testes de raciocínio, habilidades visoespaciais e memória. Sua conduta interpessoal, habilidades sociais, juízo e raciocínio permanecem intactos. A alteração da linguagem, traço característico da APP, habitualmente assume a forma de uma afasia anômica, que evolui depois para uma afasia não fluente do tipo Broca. Apesar de alguns trabalhos que descrevem uma heterogeneidade na evolução do quadro lingüístico dos pacientes com enfermidade de Alzheimer (Crystal, Horoupian, Katzman \& Jotkowitz, 1981; Joanette, Poissant \& Valdois, 1989; Joanette, Ska, Poissant \& Béland, 1992) também apresentam, entre seus sintomas iniciais, uma afasia anômica que, no entanto, evolui para uma forma transcortical sensorial ou para uma afasia de Wernicke, ambas com um forte componente de falhas de compreensão lingüística (Cardebat, Aithamon \& Puel, 1995; Habib \& Allegri, 1997).

O segundo subtipo, a demência semântica (DS), reflete um comprometimento dos sistemas cognitivos especializados no tratamento semântico (Hodges, Patterson, Oxbury \& Funnell, 1992; Hodges, Salmon \& Butters, 1988). Enquanto a memória episódica está relativamente preservada, a semântica apresenta-se profundamente alterada. Observa-se, ainda, através de recursos de neuroimagem, uma atrofia bilateral predominantemente esquerda do neocórtex temporal, inversamente à atrofia hipocâmpica - temporal medial da demência de Alzheimer (Habib \& Allegri, 1997) .

O terceiro subtipo de demência degenerativa cortical não-Alzheimer, as demências frontotemporais (DFT), tem seu início geralmente na faixa dos 40 aos 60 anos, sendo que não há predomínio de sexo. Os antecedentes familiares são observados em cerca de $50 \%$ dos casos (Kumar, Shapiro, Haxby, Grady \& Friedland, 1990; Neary e cols., 1986) e a progressão da patologia é gradual, havendo uma oscilação de 3 a 17 anos, quanto ao tempo de evolução da enfermidade (Neary e cols., 1986). Clinicamente, evidenciam-se, no estágio inicial, transtornos de personalidade, condutas anti-sociais e desinibição, sendo que, num momento posterior, aparecem transtornos da linguagem, com anomias, estereotipias e alterações na compreensão. A memória, o cálculo e a orientação visoespacial começam a se deteriorar nos estágios moderados da enfermidade. No estágio final, constata-se uma severa deterioração cognitiva global, com o surgimento de uma síndrome de acinesia progressiva e perda da linguagem (Serrano, Ranalli, Butman \& Allegri, no prelo).

Os transtornos da personalidade envolvidos na patologia são mudanças de personalidade, tais como a apatia, a desinibição sexual, uma hilaridade inapropriada, o exibicionismo e outras condutas anti-sociais (Benton, 1968). Os fenômenos de desinibição (sexual e hilaridade) são, na DFT, as manifestações clínicas mais precoces e antecedem em vários anos à deterioração cognitiva. Outras características freqüentes em sujeitos portadores da DFT são a hiperoralidade e a hipermetamorfose. A primeira se manifesta como um ato de ingestão incontrolada de qualquer alimento ou mesmo de objetos que não constituem alimento. A segunda consiste na tendência a explorar o ambiente impulsivamente, tocando em tudo. A estas características é somada a hipersexualidade, sintoma que remete a uma síndrome do tipo Kluver Bucy.

No que concerne à linguagem, observa-se uma notável diminuição da fluência, surgimento de estereotipias, ecolalias e condutas verbais reiterativas. A DFT promove uma precoce disfunção da linguagem expressiva, com a compreensão preservada até estágios avançados da patologia.

Psicologia: Reflexão e Crítica, 2001, 14(2), pp. 317-324 
O lobo pré-frontal não é uma estrutura homogênea; ele responde por múltiplas funções e se relaciona com outras estruturas, corticais e subcorticais. Esta especialização das diferentes áreas do córtex pré-frontal permite descrevê-lo basicamente em três divisões funcionais, a saber: córtex dorsolateral; córtex orbitofrontal; córtex medial. A estas divisões correspondem distintas síndromes cognitivas e de conduta (Miller e cols., 1991).

A porção dorsolateral recebe informação externa, oriunda dos sistemas sensoriais, e interna, do córtex orbitofrontal e do sistema límbico. Estes dados permitem o lobo pré-frontal monitorar o input sensorial e avaliar o significado emocional de eventos externos para iniciar uma resposta apropriada. Danos nesta área resultam em falta de resposta e comprometimento da programação motora, além de um prejuízo no automonitoramento. A região orbitofrontal está mais relacionada ao sistema límbico do que à região dorsolateral. Trata-se do representante cortical do sistema límbico. Uma síndrome orbitofrontal implica condutas anti-sociais, desinibição e inconstância emocional.

O componente medial, por sua vez, está conectado com a amígdala, o tálamo anterior e o septo. No caso de uma síndrome frontal medial, surge a acinesia como sintoma de conduta. Verifica-se um mutismo acinético, no caso de lesão bilateral, ou negligência intencional, no caso de lesão unilateral. Do ponto de vista lingüístico, o paciente pode ter uma afasia motora transcortical. A incontinência e os transtornos na marcha freqüentemente acompanham a síndrome.

Os critérios diagnósticos centrais para demência frontotemporal (DFT) são: começo insidioso e progressão gradual; deterioração precoce da conduta interpessoal e no manejo da conduta pessoal; embotamento emocional precoce; perda precoce do insight. Outros traços de ajuda diagnóstica são: declínio na higiene e no cuidado pessoal; rigidez e inflexibilidade mental; distratibilidade; hiperoralidade; condutas perseverativas; linguagem estereotipada; ecolalia; mutismo; incontinência; acinesia, rigidez e tremor. Investigações neuropsicológicas revelam deteriorização significativa nos testes do lobo frontal e ausência de amnésia, afasia ou distúrbio perceptual severo.

Pacientes com enfermidade de Alzheimer também apresentam alterações cognitivas e de comportamento. Quanto às primeiras, observa-se um prejuízo na memória, na orientação espaço-temporal, no pensamento, na concentração e na linguagem. Algumas das modificações de conduta são: o surgimento de transtornos de humor, alterações sexuais, mudança de apetite, delírios e Psicologia: Reflexão e Crítica, 2001, 14(2), pp. 317-324 alucinações, apatia e indiferença, desinibição, distúrbios de atividade psicomotora e distúrbios do sono (Mangone, 1995).

Existe uma certa dificuldade de se estabelecer um diagnóstico clínico correto para a demência frontotemporal (DFT), principalmente nos casos em que o paciente demonstra traço de apatia. Quando a doença é detectada através de um sintoma inicial de transtorno de conduta, torna-se mais clara a possibilidade de ser um caso de DFT. Não obstante, quando o sintoma é um estado de apatia e indiferença, a possibilidade de ser um caso de DFT não é tão nítida, visto que, na enfermidade de Alzheimer, também é freqüente a presença desses sintomas. Em ambos os casos encontram sintomas disexecutivos e, portanto, sua presença não é um bom critério diagnóstico. Comparando-se ambos os tipos demenciais, observam-se, entretanto, diferenças clínicas entre eles. $\mathrm{Na}$ enfermidade de Alzheimer (EA), têm-se, como indicadores iniciais, a perda de memória, a desorientação espacial e a falta de palavras. Já na DFT, o início é marcado por mudanças de personalidade e indiferença social. No que diz respeito à linguagem, o paciente com EA apresenta afasia, enquanto o paciente com DFT aparece com estereotipias, ecolalias e perseverações.

A demência tipo Alzheimer (DTA) e a demência frontotemporal (DFT) são as formas mais freqüentes de demências degenerativas primárias (Mangone, Allegri, Arizaga \& Ollari, 1997). Não obstante o termo demência frontal existir há cem anos (Pick 1892, citado por Rottenberg \& Hochberg, 1977), apenas recentemente estas entidades adquiriram características clínicas definidas. Segundo Brun e colaboradores (1994), o marco desta mudança foi o trabalho conjunto dos grupos de Lund, na Suécia, e de Manchester, na Inglaterra. Os critérios diagnósticos baseiam-se principalmente nas alterações da conduta e nos resultados obtidos por meio de neuroimagens (Brun \& cols., 1994). Entretanto, as descrições das alterações da memória de tais pacientes são escassas (Allegri, Harris, Drake, Mangone \& Arizaga, 1998; Jagust, Reed, Seab, Kramer \& Budinger, 1989; Johanson \& Hagberg 1989; Mendez e cols., 1996; Neary \& cols., 1988; Pachana e cols., 1996).

Geralmente aceita-se que a deterioração da memória é a característica predominante de início de uma DTA, enquanto que as alterações de conduta, de personalidade e de relacionamento social são as manifestações clínicas típicas mais salientes de uma DFT. Não obstante, o diagnóstico diferencial precoce é freqüentemente difícil e confuso. Por esta razão, o propósito da presente investigação foi estudar e comparar o rendimento da memória de 
pacientes com DTA, DFT e de controles normais $(\mathrm{CON})$, a fim de estabelecer os perfis correspondentes.

\section{Método}

\section{Participantes}

Do arquivo de nosso centro (CEMIC) foram selecionados 20 pacientes que cumpriam os critérios clínicos diagnósticos de Lund e Manchester (Brun e cols., 1994) de DFT e mostravam uma tomografia por emissão de fóton único cerebral (SPECT) apresentando hipoperfusão frontotemporal (como por exemplo, observa-se na Figura 1, os resultados da Ressonância Magnética Nuclear e na Figura 2, os resultados do SPECT do paciente JL) e 20 outros pacientes que cumpriam os critérios de NINCDS ADRDA para DTA (McKhan e cols., 1984) e exibiam uma hipoperfusão predominantemente temporo-parietal. Vinte sujeitos controles foram escolhidos por emparelhamento de idade e escolaridade com os pacientes, na população em geral. Foram excluídos todos aqueles sujeitos com alterações neurológicas ou psiquiátricas que poderiam causar outro tipo de demência, bem como pacientes com diagnóstico compatível com Afasia Progressiva Primária ou Demência Semântica (Habib \& Allegri, 1997).
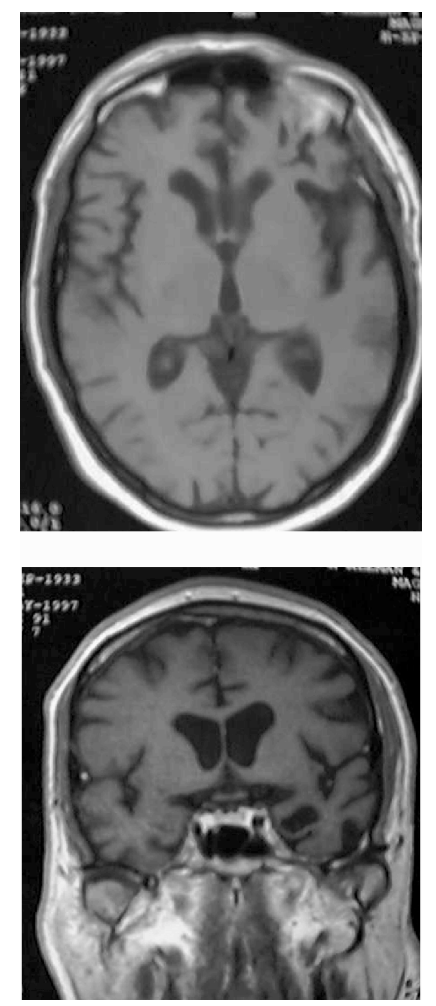

Figura 1. Ressonância magnética cerebral em um dos pacientes com demência frontotemporal (JL). Nas imagens observa-se uma atrofia frontotemporal de predomínio direito.
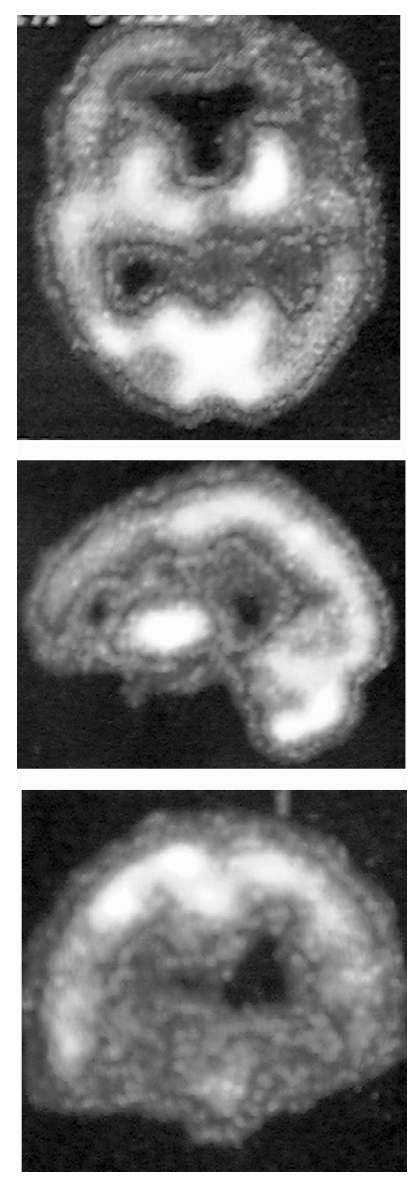

Figura 2. SPECT cerebral em um dos pacientes com DFT (LJ). Nas imagens observa-se uma hipoperfusão frontotemporal bilateral de predomínio direito.

\section{Procedimentos}

Todos os pacientes foram avaliados neurológica, neuropsicológica e neuropsiquiatricamente, incluindo o teste Mini-mental (Folstein, Folstein \& McHugh, 1975). Também foram submetidos a uma série de exames incluindo dosagem de hormônios tireóideos, dosagem de vitamina B12, etc. - assim como à investigação por neuroimagens (Ressonância Magnética Nuclear e SPECT cerebral).

A avaliação da memória incluiu investigação das seguintes memórias:

Memória Subjetiva: a fim de estudar a "queixa de dificuldade de memória que reportavam os próprios pacientes" utilizou-se uma adaptação do Questionário de Memória Subjetiva (CMS) de McNair e Kahn (1983, adaptado para o espanhol por Allegri e cols., 1999). Este questionário é composto de 17 perguntas sobre diferentes situações que caracterizam falhas de memória retrospectiva, prospectiva, semântica e visual. Por exemplo: "Você sente que tem uma palavra na ponta da língua, mas não consegue lembrá-la?” E "Você vai a um lugar e esquece do que ia fazer ali?". As 
respostas são registradas em escala de Likert de cinco pontos, cujas opções vão de "raramente" a "quase nunca".

Memória episódica: a memória episódica foi avaliada através de uma prova de aprendizagem de uma lista de palavras (Allegri, Harris \& Arizaga, 1998; Allegri, Harris, Feldman, Taragano \& Paz, 1998). Esta prova consiste em 12 palavras não relacionadas, pertencentes a 12 categorias semânticas distintas, de grande imageabilidade, ou seja, palavras que denotam objetos concretos, e de freqüência média, que não se correspondem com os exemplares típicos representativos de uma certa categoria. A administração desta prova inicia-se com a leitura da lista de palavras pelo examinador. Depois da leitura, o sujeito deve relatar as palavras que recorda, sem precisar respeitar a ordem. Este procedimento repete-se mais duas vezes, por três ensaios (AS1, AS2, AS3), a chamada fase de "aprendizagem serial". Depois de um intervalo de dez minutos, durante o qual se realizam outras provas cognitivas (por exemplo, o span de dígitos), avalia-se a "recordação livre deferida" (RLD), solicitando-se ao sujeito que diga as palavras que recorda espontaneamente da lista aprendida previamente. Para aquelas palavras que não foram evocadas livremente efetua-se uma prova de reconhecimento por escolha múltipla (1 alvo e 3 distratores).

Para a presente investigação, foram considerados os seguintes parâmetros:

1. Total de respostas corretas nas seguintes etapas:

1.1. terceiro ensaio da aprendizagem serial (AS),

1.2. recordação livre deferida (RLD),

1.3. reconhecimento por escolha múltipla $(\mathrm{Re})$

2. Índice de esquecimento: $\mathrm{O}$ índice de esquecimento quantifica o grau de perda da informação adquirida durante a aprendizagem. Ele é obtido através do quociente entre a diferença entre o escore adquirido na aprendizagem ( $\mathrm{AL}=$ Aprendizagem da Lista: valor máximo alcançado nos ensaios) e o escore da recordação livre deferida (AL-RLD).

3. Índice de recuperação: o índice estabelece o grau de recuperação facilitada pela escolha múltipla daquelas palavras que não foram evocadas espontaneamente. Obtém-se, pelo quociente entre a diferença do recuperado por escolha múltipla e o correspondente à recordação livre deferida (Re-RLD).

Memória semântica: para estudar o componente semântico, utilizaram-se o teste de denominação por confrontação visual de Boston (Kaplan, Goodglass \& Weintraub, 1986), versão Buenos Aires (Allegri, Mangone, Rymberg, Fernandez \& Taragano, 1997), e o subteste de vocabulário do WAIS (Wechsler, 1988).

\section{Resultados}

A análise estatística foi realizada através de uma análise de variância (ANOVA) para a comparação entre os grupos. Para seu processamento, utilizou-se o pacote estatístico BMDP90 (Dixon, 1990). Os três grupos encontram-se emparelhados por idade e escolaridade (ver Tabela 1). Não se observam diferenças significativas entre os dois grupos de demências quanto ao nível intelectual global (WAIS) e quanto ao nível de deterioração global (Escala de Deterioração Global de Reisberg, Ferris, DeLeon \& Crook, 1982)

Os resultados dos testes de memória podem ser observados na Tabela 2. As pontuações de ambos os grupos de pacientes, na grande maioria das provas, foram significativamente inferiores àquelas alcançadas pelo grupo controle.

Tabela 1. Dados Clínicos e Demográficos

\begin{tabular}{|c|c|c|c|c|c|}
\hline & \multirow[b]{2}{*}{ DFT } & \multirow[b]{2}{*}{ DTA } & \multirow[b]{2}{*}{$\mathrm{CON}$} & \multicolumn{2}{|c|}{ ANOVA } \\
\hline & & & & $F$ & $p$ \\
\hline$N$ de pacientes & 20 & 20 & 20 & & \\
\hline Idade (anos) & $65,2(10,1)$ & $68,4 \quad(8,0)$ & $65,9 \quad(8,6)$ & 0,9 & ns \\
\hline Educação (anos) & $12,6 \quad(3,8)$ & $13,0 \quad(4,4)$ & $12,9 \quad(4,1)$ & 0,4 & ns \\
\hline CIT (WAIS) & $96,2(12,9)$ & $83,5(14,1)$ & $114,0(12,2)$ & $\# 4,7$ & ns \\
\hline EDG & $3,7 \quad(0,4)$ & $3,9 \quad(0,5)$ & - & \# 0,8 & ns \\
\hline
\end{tabular}

Referências: CIT = coeficiente intelectual total do WAIS (11); EDG = Escala de deterioração global (19); \# = DFT vs DTA; ns = não significativo. 
Os pacientes com DFT e com DTA não apresentaram diferenças significativas na aprendizagem nem na recordação serial. Quanto à prova de reconhecimento por escolha múltipla, o grupo com DFT obteve uma melhor recordação do que o grupo com DTA. Este mesmo efeito observa-

\section{Discussão}

A avaliação neuropsicológica é uma ferramenta básica para o diagnóstico das demências incipientes (Allegri, 1995; Allegri, Harris \& Drake, 2000). Sua utilização para

Tabela 2. Resultados da avaliação de memória

\begin{tabular}{|c|c|c|c|c|c|}
\hline & & & & & \\
\hline & DFT & DTA & Controles & $F$ & $p$ \\
\hline Memória Episódica & & & & & \\
\hline Aprendizagem serial (14) & $5,4(1,4)$ & $4,0 \quad(2,0)$ & $9,8(1,4)$ & 3,2 & ns \\
\hline Recordação serial (14) & $2,6(2,5)$ & $2,1 \quad(2,2)$ & $9,3(2,0)$ & 4,0 & ns \\
\hline Reconhecimento (14) & $8,7(2,2)$ & $5,0 \quad(4,0)$ & $11,4(1,3)$ & 4,4 & $<0,05$ \\
\hline Índice de esquecimento & 0,44 & 0,47 & 0,005 & & ns \\
\hline Índice de Recuperação & 0,70 & 0,58 & 0,18 & 6,4 & $<0,01$ \\
\hline Memória Semântica & & & & & \\
\hline Denominação (16) & $45,8(9,9)$ & $34,1(14,1)$ & $51,9(6,1)$ & 9,2 & $<0,005$ \\
\hline Vocabulário (11) & $10,1(4,1)$ & $9,1 \quad(4,2)$ & $13,3(2,1)$ & 0,8 & ns \\
\hline Memória Subjetiva & & & & & \\
\hline Queixa subjetiva & $2,4(2,1)$ & $5,3 \quad(2,8)$ & $4,3(1,4)$ & 12,7 & $<0,001$ \\
\hline
\end{tabular}

Referências: os valores estão expressos em média (desvio-padrão), $(\#)=$ ANOVA de DFT vs DTA, ns= não significativo

se no índice de esquecimento, similar em ambas as populações, mas diferente no índice de recuperação, sendo este superior no grupo com DFT. Isso demonstra que os pacientes com DTA perdem a informação que adquiriram, pois não a registram adequadamente. Contrariamente, os pacientes com DFT não conseguem recuperar espontaneamente a informação, mas o fazem com a facilitação por escolha múltipla, o que implica uma falha no mecanismo de recuperação e não em uma perda de registro, como no caso das DTA. A prova de memória semântica estava significativamente pior no grupo com DTA do que no grupo com DFT.

Com relação à "queixa subjetiva dos pacientes quanto a seus esquecimentos", os pacientes com DTA queixaramse mais (ainda que sem diferença estatisticamente significativa) do que os controles, e aqueles com DFT queixaram-se significativamente menos, provavelmente devido a sua maior anosognosia, ou seja, uma falha patológica no reconhecimento de suas deficiências.

Para estudar as distintas partes da memória (arquivo e recuperação) nos testes de aprendizagem serial, recuperação livre e recuperação facilitada (reco-nhecimento), os resultados das provas de memória foram comparados mediante o cálculo da pontuação Z (ver Figura 3). o diagnóstico diferencial entre as formas de demência degenerativa tem sido subestimada e escassos autores ocuparam-se com esta temática (Allegri e cols., 1998, 2000; Jagust e cols., 1989; Johanson \& Hagberg, 1989; Mendez e cols., 1996; Neary e cols., 1986; Pachana e cols., 1996), apesar da dificuldade no diagnóstico diferencial entre as

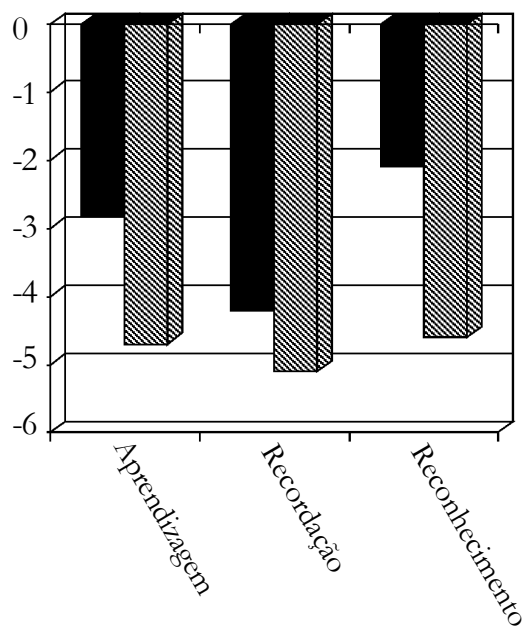

Figura 3. Perfis de memória diferenciais entre demências frontotemporais (DFT) e demências de tipo Alzheimer (DTA), segundo escores padronizados $\mathrm{Z}$ para cada prova 
diversas formas de demência apontada em diferentes trabalhos (Pachana e cols., 1996).

Nos últimos anos, medicações específicas para a enfermidade de Alzheimer, como os anticolinesterásicos tipo Tacrina, Donepezil, Rivastigmine e Galantamina começaram a ser desenvolvidas (Schneider \& Forette, 1996). Isso obriga cada vez mais a realização de um diagnóstico precoce desta patologia e a sua diferenciação quanto à DFT.

Os resultados das avaliações neuropsicológicas de ambos os tipos de demências estão significativamente abaixo dos resultados obtidos pelos sujeitos-controle. Apesar do conceito clássico de falta de distúrbio de memória nas formas incipientes de DFT (Cummings \& Benson, 1992), em nossa população, a falha mnemônica era freqüente em ambas demências (Allegri e cols., 1999). Este achado é controvertido na literatura: alguns autores encontraram alterações de memória (Allegri e cols., 1998; Johanson \& Hagberg, 1989; Neary e cols., 1986) e outros não (Jagust e cols., 1989). Entretanto, em nosso trabalho, foram encontrados perfis mnemônicos diferentes. Comparando os resultados dos testes neuropsicológicos obtidos, ambos os grupos de pacientes falhavam na aprendizagem da lista de palavras, tanto na recordação livre como na lista diferida de palavras, mas os pacientes com DFT melhoravam significativamente quando havia uma facilitação.

Esses resultados podem ser explicados através de modelos que explicam o funcionamento e estruturas da memória. Ela pode ser concebida como um fluxo de informação entre diferentes arquivos interrelacionados. Assim, a informação que ingressa passa por diferentes estágios: aquisição, consolidação e recuperação (Tamaroff \& Allegri, 1995). De acordo com esta perspectiva, os sujeitos com DFT teriam uma dificuldade na recuperação (por exemplo: inabilidade do sistema de memória para localizar o traço mnemônico). A aquisição e a consolidação estariam conservadas; ainda que o sujeito não recordasse espontaneamente, a informação arquivada seria acessada quando houvesse uma facilitação, como sucede com a escolha múltipla. Esta característica é similar à referida nos quadros subcorticais, como a enfermidade de Parkinson (Allegri e cols., 1998), que seria melhor denominada "esquecimentos" e não "amnésia". Por outro lado, os pacientes com DTA têm dificuldades na aquisição e na consolidação da informação por falhas de codificação. Conseqüentemente, a informação não é transferida da memória a curto prazo à memória a longo prazo (característica "amnésica”).

Os sujeitos com DFT tiveram resultados significativamente melhores que aqueles com DTA no subteste de denominação. Isso demonstra um menor distúrbio das funções semânticas nas DFT, onde não são encontradas as formas afásicas e a alteração precoce na EA, como mostraram Mendez e colaboradores (1996) e Pachana e colaboradores (1996).

Concluindo, o perfil mnemônico diferencial da demência frontotemporal com relação à enfermidade de Alzheimer permite afirmar que a presença de uma boa recuperação nas provas de memória, como a de reconhecimento, seria um dos traços cognitivos diferenciais. A partir dos presentes resultados, podemos concluir que os perfis mnemônicos em sujeitos com DTA e DFT com similar nível de compromisso cognitivo são diferentes. Assim, deveríamos supor que existe uma dicotomia entre as demências corticais de perfis mnemônicos cortical posterior na DTA e cortical anterior na DFT: a primeira é caracterizada por um comprometimento na armazenagem da informação e a segunda por um comprometimento apenas na etapa de recuperação.

\section{Referências}

Allegri, R. F. (1995). Estudios Complementarios para el Diagnóstico de la Enfermedad de Alzheimer. Em Mangone, C. A., Allegri, R. F., Arizaga, L., Famulari A.L., Fustinoni O, Ollari J.A., \& Sica REP. Enfermedad de Alzheimer: Enfoque actual. Buenos Aires: Argentum.

Allegri R. F., Harris P. \& Arizaga R. (1998). Different cognitive profiles on memory in Parkinson's Disease and Alzheimer's Disease. Em Y. Hanin, A. Fisher, M. Yoshida (Orgs.), Progress in Alzheimer's and Parkinson's diseases (pp. 265-270). New York: Plenum Press.

Allegri, R. F., Harris, P. \& Drake, M. (2000). Evaluación neuropsicológica en las demencias. Revista Neurologica Argentina, 24, 11-15.

Allegri R. F., Harris P., Drake M., Mangone C., A., \& Arizaga R. L. (1998). Memoria en la enfermedad de Alzheimer y en la enfermedad de Parkinson: Estudio del aprendizaje serial, recuerdo com claves y reconocimiento. Revista Neurologica Argentina, 23, 19-24.

Allegri, R. F., Harris, P., Feldman, M., Taragano, F. \& Paz, J. (1998). Perfiles cognitivos diferenciales entre la demencia frontotemporal y la demencia tipo Alzheimer. Revista de Neurología, 27, 463-466.

Allegri, R. F., Mangone, C. A., Rymberg, S., Fernandez A. \& Taragano, F. E. (1997). Spanish Boston Naming Test Norms. The Clinical Neuropsychologist, 11(4), 416-420.

Allegri, R. F., Taragano, F. E., Feldman, M., Harris, P., Elorza, P., Tufró, G., Martelli, M., Sánchez, V., Goscilo, C., Nudelman, A. \& Tamaroff, L. (1999). La "queja de pérdida de memoria" en el diagnóstico de las demencias. Prensa Medica Argentina, 86, 670-677.

Almeida, O. P. \& Nitrini, R. (1995). Demência. São Paulo: Fundo Editorial BYK.

Benton, A. L. (1968). Differential behavioral effects in frontal lobe disease. Neuropsychologia, 5, 53-60.

Brun, A., Englund, B., Gustafson, L., Passant, U., Mann, D. M. A., Neary, D., \& Snowden, J. S. (1994). Clinical and neuropathological criteria for frontotemporal dementia. Journal of Neurological and Neurosurgical Psychiatry, 57, 416-418.

Cardebat, D., Aithamon, B. \& Puel, M. (1995). Troubles du langage dans les démences de type Alzheimer. Em F. Eustache \& A. Agniel (Orgs.), Neuropsychologie clinique des démences: évaluations et prises en charge (pp. 183198). Marseille: Solal.

Crystal, H. A., Horoupian, D. S., Katzman, R. \& Jotkowitz, S. (1981) Biopsyproved Alzheimer Disease presenting as right parietal lobe syndrome. Annais of Neurology, 12, 186-188. 
Cummings, J. L. \& Benson, D. F. (1992). Dementia: A clinical approach. (2a. ed.). Stoneham: Butterworth Heinemann.

Dewer, B. (1995). Interêt de la dissociation entre démences corticales et démences sous-corticales. Em F. Eustache \& A. Agniel (Orgs.), Neuropsychologie clinique des démences: Évaluations et prises en charge (pp. 123136). Marseille: Solal.

Dixon, W. J. (1990). BMDP Statistical Software. Los Angeles: University of California (UCLA), PC 90.

Duyckaerts, C., He, E., Seilhean, D. \& Hauw, J-J. (1995). Démences frontales et atrophies focales: Données neuro-pathologiques. Em F. Eustache Données \& A. Agniel (Orgs.), Neuropsychologie Clinique des Démences: Évaluations et prises en charge (pp. 63-70). Marseille: Solal.

Folstein, M. F., Folstein, S. E. \& McHugh, P. R. (1975). "Mini-mental State”: A practical method for grading the cognitive state of patients for the clinician. Journal of Psychiatric Research, 12, 189-198.

Habib, M. \& Allegri, R. F. (1997). Síndromes neuropsicologicos focales. Em C. A. Mangone, R. F. Allegri, R. L. Arizaga \& J. A. Ollari (Orgs.), Demencia: Aproximación multidisciplinaria. Buenos Aires: Sagitario.

Hodges, J. R., Patterson, K., Oxbury, S. \& Funnell, E. (1992). Semantic dementia. Progressive fluent aphasia with temporal lobe atrophy. Cognition, 115, 1783-1806.

Hodges, J. R., Salmon, D. P. \& Butters, H. (1988). Semantic memory impairment in Alzheimer Disease: Failure of access or degraded knowledge? Neuropsychologia, 30(4), 301-314.

Jagust, W. L., Reed, B. R., Seab, J. P., Kramer, J. H. \& Budinger, T. F. (1989). Clinical-physiologic correlates of Alzheimer's disease and frontal lobe dementia. American Journal of Physiologic Imaging, 4, 89-96.

Joanette, Y., Poissant, A. \& Valdois, S. (1989). Neuropsychological dissociations in dementia of the Alzheimer type: A multiple single case study. Journal of Clinical and Experimental Neuropsychology, 11, 91-97.

Joanette, Y., Ska, S., Poissant A. \& Béland, R. (1992). Neuropsychological aspects of Alzheimer's Disease: Evidence for inter- and intra-function heterogeneity. Em F. Boller, F. Forette, Z. Khachaturian, M. Poncet \& Y. Christen (Orgs.), Heterogeneity of Alzheimer's Disease (pp. 33-42). New York: Springer-Verlag.

Johanson, A. \& Hagberg, B. (1989). Psychometric characteristics in patients with frontal lobe degeneration of non-Alzheimer type. Archives of Gerontological Geriatry, 8, 129-137.

Kaplan, E., Goodglass, H. \& Weintraub, S. (1986). Test de Vocabulario de Boston. Em H. Goodglass \& E. Kaplan (Orgs.), La evaluación de la afasia (pp. 186-190). Madrid: Panamericana.

Kumar, A., Shapiro, M. B., Haxby, J. V., Grady, C. L. \& Friedland, R. P. (1990). Cerebral metabolic and cognitive studies in dementia with frontal lobe behavioral features. Journal of Psychiatric Research, 24, 97-109.

Mangone, C. A. (1995). Sindromes demenciales. Em L. Tamaroff \& R. F. Allegri (Orgs.), Introduccion a la neuropsicologia clinica (pp. 217-232). Buenos Aires: Argentum.
Mangone, C. A., Allegri, R. F., Arizaga, R. L. \& Ollari, J. A. (1997). Demencia: Aproximación multidisciplinaria. Buenos Aires: Sagitario.

McKhann, G., Drachman, D., Folstein, M., Katzman, R., Price, D. \& Stadlan, E. M. (1984). Clinical diagnosis of Alzheimer's Disease: Report of NINCDS-ADRDA Work Group under the auspices of Department of Health and Human Service Task Force on Alzheimer 's disease. Neurology, 34, 939-944.

McNair, M. \& Kahn, R. (1983). Self-assessment of cognitive deficits. Em T. Crook, S. Ferris \& R. Bartus (Orgs.), Assessment in geriatric psychopharmacology (pp. 137-144). New Canaan: Powey.

Mendez, M. F., Cherrier, M., Perryman, K. M., Pachana, N., Miller, B. L. \& Cummings, J. L. (1996). Frontotemporal dementia versus Alzheimer's Disease: Differential cognitive features. Neurology, 47, 1189-1194.

Mesulam, M. M. (1982). Slowly progressive dysphasia without generalized dementia. Annual of Neurology, 11, 592-598.

Miller, B. L., Cummings, J. L., Meyer, J. V., Bonne, K., Mehringer, C. M., Lesser, M., I \& Mena, I. (1991). Frontal lobe degeneration: Clinical neuropsychological and spect characteristics. Neurology, 41, 1374-1382.

Mortimer, J. A. (1983). Alzheimer's Disease and Senile Dementia: Prevalence and incidence. Em B. Reisberg (Orgs.), Alz̧heimer's Disease. (pp.54-79). New York: Free Press.

Neary, D., Snowden, J. S., Bowen, D. M., Sims, N. R., Mann, D. M. A., Benton, J. S., Northen, B., Yates, P. O. \& Davison, N. A. (1986). Neuropsychological syndromes in presenile dementia due to cerebral atrophy. Journal of Neurological and Neurosurgical Psychiatry, 49, 163-174.

Pachana, N. A., Bonne, K. B., Miller, B. L., Cummings, J. L. \& Berman, N. (1996). Comparison of neuropsychological functioning in Alzheimer's Disease and frontotemporal dementia. Journal of International Neuropsychological Society, 2, 505-510.

Reisberg, B., Ferris, S. H., DeLeon, M. J. \& Crook, T. (1982). The global deterioration scale of assessment of primary degenerative dementia. British Journal of Psychiatry, 139, 1136-1139.

Rottenberg, D. A. \& Hochberg, F. E. (1977). Neurological classics in modern translation. New York: Hafner Press.

Schneider, L. \& Forette, F. (1996). Alzheimer's Disease symptomatic drugs: Tacrine. Em. M. S. Gauthier (Org.), Clinical diagnosis and management of Alzheimer's Disease (pp. 221-238). UK: Dunitz.

Serrano, C., Ranalli, C. G., Butman, J. \& Allegri, R. F. (no prelo). Demencias frontotemporales. Buenos Aires: Vertex.

Tamaroff, L. \& Allegri, R. F. (1995). Introducción a la neuropsicología clínica. Buenos Aires: Argentum.

Wechsler, D. (1988). Test de Inteligência para adultos (WAIS). Buenos Aires: Paidos.

Recebido: 11/01/2001

Revisado: 12/03/2001

Aceite final: 23/05/2001

Sobre os autores:

Ricardo F. Allegri é Médico Neurologista. Investigador Clínico do CONICET (Conselho Nacional de Investigações Científicas e Tecnológicas) Chefe do Serviço de Neuropsicologia do CEMIC Hospital associado à Universidade de Buenos Aires. Docente Autorizado - Neurologia - Universidade de Buenos Aires. Diretor da Revista Neurológica Argentina.

Paula Harris é Psicóloga. Integrante do CONICET (Conselho Nacional de Investigações Científicas e Tecnológicas). Neuropsicóloga do CEMIC - Hospital associado à Universidade de Buenos Aires.

Cecília Serrano é Médica Neurologista, Felow em Neuropsicología do CEMIC - Hospital

associado à Universidade de Buenos Aires.

Nélson Delavald é aluno do Curso de Psicologia do Instituto de Psicologia da Universidade

Federal do Rio Grande do Sul. 